\title{
Correction to: Liver fibrosis assessment with multiphasic dual-energy CT: diagnostic performance of iodine uptake parameters
}

\author{
Yasunori Nagayama ${ }^{1}$ (D) Yuki Kato ${ }^{1} \cdot$ Taihei Inoue $^{1} \cdot$ Takeshi Nakaura $^{1} \cdot$ Seitaro Oda ${ }^{1} \cdot$ Masafumi Kidoh $^{1}$. \\ Osamu Ikeda ${ }^{1} \cdot$ Toshinori Hirai $^{1}$
}

Published online: 11 May 2021

(C) European Society of Radiology 2021

\section{Correction to: European Radiology} https://doi.org/10.1007/s00330-021-07706-2

The original version of this article, published on 25 March 2021, unfortunately contained a mistake. The following correction has therefore been made in the original: In Table 4 , the values for $\Delta \mathrm{HU}_{\text {liver_DP }}(\mathrm{HU})$ in F0-2 and F0-3 groups were incorrect. The corrected table is given below. The original article has been corrected.

The online version of the original article can be found at https://oi.org/ 10.1007/s00330-021-07706-2

Yasunori Nagayama

y.nagayama1980@gmail.com

1 Department of Diagnostic Radiology, Graduate School of Medical Sciences, Kumamoto University, 1-1-1, Honjo, Chuo-ku,

Kumamoto 860-8556, Japan 


\begin{tabular}{|c|c|c|c|c|c|c|c|c|c|}
\hline \multirow[t]{2}{*}{ Parameters } & \multicolumn{2}{|l|}{ F stage } & \multirow[t]{2}{*}{$p$ value } & \multicolumn{2}{|l|}{ F stage } & \multirow[t]{2}{*}{$p$ value } & \multicolumn{2}{|l|}{ F stage } & \multirow[t]{2}{*}{$p$ value } \\
\hline & $\begin{array}{l}\text { F0-1 } \\
(n=47)\end{array}$ & $\begin{array}{l}\mathrm{F} 2-4 \\
(n=70)\end{array}$ & & $\begin{array}{l}\text { F0-2 } \\
(n=66)\end{array}$ & $\begin{array}{l}\mathrm{F} 3-4 \\
(n=51)\end{array}$ & & $\begin{array}{l}\mathrm{F} 0-3 \\
(n=88)\end{array}$ & $\begin{array}{l}\mathrm{F} 4 \\
(n=29)\end{array}$ & \\
\hline Age (years) & $67 \pm 12$ & $69 \pm 12$ & 0.33 & $68 \pm 11$ & $68 \pm 13$ & 0.78 & $67.9 \pm 12.4$ & $68.9 \pm 9.9$ & 0.64 \\
\hline Men & $36(76.6)$ & $47(67.1)$ & 0.30 & $50(75.8)$ & $33(64.7)$ & 0.22 & $67(76.1)$ & $16(55.2)$ & 0.04 \\
\hline Body mass index $\left(\mathrm{kg} / \mathrm{m}^{2}\right)$ & $23.8 \pm 3.3$ & $24.6 \pm 4.0$ & 0.28 & $23.7 \pm 3.2$ & $25.0 \pm 4.2$ & 0.06 & $23.9 \pm 3.6$ & $25.5 \pm 4.0$ & 0.06 \\
\hline Hematocrit $(\%)$ & $37.5 \pm 6.1$ & $37.7 \pm 5.3$ & 0.84 & $37.5 \pm 5.7$ & $37.8 \pm 5.6$ & 0.81 & $37.9 \pm 6.6$ & $37.0 \pm 5.6$ & 0.47 \\
\hline \multicolumn{10}{|l|}{ SSDE (mGy) } \\
\hline Pre-contrast & $15.6 \pm 2.2$ & $15.8 \pm 3.5$ & 0.68 & $15.5 \pm 2.1$ & $16.0 \pm 3.8$ & 0.38 & $16.1 \pm 3.8$ & $15.6 \pm 2.7$ & 0.53 \\
\hline PVP & $17.4 \pm 2.5$ & $17.6 \pm 3.2$ & 0.70 & $17.3 \pm 2.3$ & $17.7 \pm 3.6$ & 0.42 & $18.0 \pm 3.5$ & $17.3 \pm 2.7$ & 0.36 \\
\hline $\mathrm{DP}$ & $17.0 \pm 2.8$ & $17.1 \pm 3.3$ & 0.90 & $16.8 \pm 2.6$ & $17.4 \pm 3.6$ & 0.18 & $17.5 \pm 3.6$ & $16.9 \pm 2.9$ & 0.42 \\
\hline \multicolumn{10}{|l|}{$\Delta \mathrm{HU}$ parameters } \\
\hline$\Delta \mathrm{HU}_{\text {liver PVP }}(\mathrm{HU})$ & $62.4 \pm 10.6$ & $60.9 \pm 10.2$ & 0.44 & $62.3 \pm 10.4$ & $60.5 \pm 10.3$ & 0.33 & $61.8 \pm 10.3$ & $60.6 \pm 10.6$ & 0.59 \\
\hline$\Delta \mathrm{HU}_{\text {liver DP }}(\mathrm{HU})$ & $36.8 \pm 5.8$ & $40.4 \pm 6.4$ & 0.001 & $37.2 \pm 6.1$ & $41.3 \pm 6.1$ & $<0.001$ & $37.9 \pm 6.3$ & $42.2 \pm 5.6$ & 0.001 \\
\hline $\mathrm{ECV}_{\Delta \mathrm{HU}}(\%)$ & $26.8 \pm 3.7$ & $29.6 \pm 5.1$ & $<0.001$ & $26.8 \pm 3.7$ & $30.7 \pm 5.3$ & $<0.001$ & $27.7 \pm 4.5$ & $31.0 \pm 4.8$ & 0.004 \\
\hline $\operatorname{IWR}_{\Delta H U}(\%)$ & $40.7 \pm 5.0$ & $32.9 \pm 9.7$ & $<0.001$ & $40.2 \pm 5.0$ & $30.7 \pm 10.1$ & $<0.001$ & $38.3 \pm 7.4$ & $29.3 \pm 10.2$ & $<0.001$ \\
\hline \multicolumn{10}{|l|}{ ID parameters } \\
\hline $\mathrm{ID}_{\text {liver_PVP }}(\mathrm{mg} / \mathrm{mL})$ & $2.43 \pm 0.42$ & $2.32 \pm 0.41$ & 0.18 & $2.43 \pm 0.41$ & $2.28 \pm 0.40$ & 0.06 & $2.39 \pm 0.42$ & $2.29 \pm 0.40$ & 0.23 \\
\hline $\mathrm{ID}_{\text {liver_DP }}(\mathrm{mg} / \mathrm{mL})$ & $1.42 \pm 0.23$ & $1.55 \pm 0.24$ & 0.003 & $1.44 \pm 0.24$ & $1.58 \pm 0.23$ & $<0.001$ & $1.46 \pm 0.24$ & $1.60 \pm 0.23$ & 0.008 \\
\hline $\operatorname{ECV}_{\mathrm{ID}}(\%)$ & $24.0 \pm 3.7$ & $27.0 \pm 4.9$ & $<0.001$ & $24.1 \pm 3.6$ & $27.9 \pm 5.1$ & $<0.001$ & $25.0 \pm 4.4$ & $28.1 \pm 4.8$ & 0.002 \\
\hline $\operatorname{IWR}_{\mathrm{ID}}(\%)$ & $41.8 \pm 4.6$ & $32.4 \pm 9.1$ & $<0.001$ & $40.6 \pm 4.6$ & $30.1 \pm 9.4$ & $<0.001$ & $38.3 \pm 7.3$ & $29.1 \pm 9.5$ & $<0.001$ \\
\hline \multicolumn{10}{|l|}{ Serum fibrosis markers } \\
\hline APRI & $0.39 \pm 0.27$ & $0.93 \pm 1.01$ & $<0.001$ & $0.43 \pm 0.30$ & $1.09 \pm 1.13$ & $<0.001$ & $0.56 \pm 0.78$ & $1.17 \pm 0.85$ & 0.001 \\
\hline FIB-4 & $2.17 \pm 1.09$ & $4.13 \pm 2.42$ & $<0.001$ & $2.34 \pm 1.16$ & $4.65 \pm 2.56$ & $<0.001$ & $2.64 \pm 1.8$ & $5.18 \pm 2.33$ & $<0.001$ \\
\hline
\end{tabular}

Data are presented as mean \pm standard deviation or number $(\%)$

$A P R I$ alanine aminotransferase-to-platelet ratio index, $D P$ delayed phase, FIB-4 fibrosis-4 index, $I D$ iodine density, $P V P$ portal-venous phase, SSDE size-specific dose estimate

Publisher's note Springer Nature remains neutral with regard to jurisdictional claims in published maps and institutional affiliations. 